\title{
Vitamin D: Ten Beliefs
}

\author{
G. Michael Allan, $M D^{7}$, James McCormack, PharmD ${ }^{2}$, and Christina Korownyk, $M D^{7}$ \\ ${ }^{1}$ Evidence-Based Medicine, Department of Family Medicine - Research Program, University of Alberta, Edmonton, AB, Canada; ${ }^{2}$ Faculty of \\ Pharmaceutical Sciences, University of British Columbia, Vancouver, BC, Canada.
}

$\mathrm{J}$ Gen Intern Med 31(11): 1275

DOI: $10.1007 / \mathrm{s} 11606-016-3699-\mathrm{x}$

(c) Society of General Internal Medicine 2016

$\mathrm{D}$ r. Grant raises some interesting points regarding our publication, "Vitamin D: A Narrative Review Examining the Evidence for Ten Beliefs." First, he recommends that nutrients should be viewed through a different research lens than pharmaceutical drugs. Serum levels should be done, only those with low levels should be randomized, vitamin D should be given sufficiently to raise levels to certain targets and then differences in outcomes assessed. This is certainly an attractive hypothesis and worthy of testing. However, in the absence of high quality randomized trials, this suggestion remains a hypothesis. Until proven, we have to rely on the best available evidence, such as that presented in our review.

Second, Dr. Grant presents evidence of cancer risk associated with lower vitamin D levels, a point made in our paper. ${ }^{1}$ However, this evidence is observational and any potential benefits resulting from Vitamin D supplementation are only an extrapolation from this lower-level evidence. Unfortunately, a cancer benefit from Vitamin D supplements is not supported by the available higher-level evidence, which suggests future research on cancer mortality is unlikely to yield meaningful change in the estimate of no effect. ${ }^{2}$

Third, to support the belief that individuals with lower serum levels are more likely to reap benefits from vitamin supplementation, Dr. Grant references a single study that demonstrated infection prevention with supplementation for those with low Vitamin D levels. We too had raised this potential important linkage in our paper, ${ }^{1}$ but noted the results cannot be applied to all developing countries. A large trial in Kabul Afghanistan did not see any reduction in pneumonia when infants were provided with vitamin D supplementation, despite vitamin $\mathrm{D}$ levels being roughly $50 \mathrm{nmol} / \mathrm{L}$ or higher in the treated arm, while levels in the untreated arm were lower by roughly $20 \mathrm{nmol} / \mathrm{L}$ or more at most time points (Vitamin D levels estimated from Fig. 3). ${ }^{3}$

Overall, evidence for the health benefits of vitamin D supplementation is unconvincing. Further research is needed, but additional observational studies and small, low-quality trials will only muddy the waters. From here on in, Vitamin D research should emphasize high-quality randomized, controlled trials, particularly in individuals with lower serum concentrations.

Corresponding Author: G. Michael Allan, MD; Evidence-Based Medicine, Department of Family Medicine - Research ProgramUniversity of Alberta, 6-10 University Terrace, Edmonton, AB T6G 2T4, Canada (e-mail: michael.allan@ualberta.ca).

\section{REFERENCES}

1. Allan GM, Cranston L, Lindblad A, McCormack J, Kolber MR, Garrison S, Korownyk C. Vitamin D: A Narrative Review Examining the Evidence for Ten Beliefs. J Gen Intern Med. doi: 10.1007/s11606-016-3645-y.

2. Bolland MJ, Grey A, Gamble GD, Reid IR. The effect of vitamin D supplementation on skeletal, vascular, or cancer outcomes: a trial sequential meta-analysis. Lancet Diabetes Endocrinol. 2014;2(4):307-320.

3. Manaseki-Holland S, Maroof Z, Bruce $\mathbf{J}$, et al. Effect on the incidence of pneumonia of vitamin D supplementation by quarterly bolus dose to infants in Kabul: a randomised controlled superiority trial. Lancet. 2012;379(9824):1419-27.

Published online April 15, 2016 